\title{
Relaxation dynamics of magic clusters
}

\author{
Y. D. Kim,* M. Niemietz, P. Gerhardt, F. v. Gynz-Rekowski, and G. Ganteför \\ Department of Physics, University of Konstanz, D-78457 Konstanz, Germany
}

\begin{abstract}
Using femtosecond time-resolved two-photon photoemission spectroscopy, relaxation rates of the optically excited states in $\mathrm{Al}_{13}^{-}, \mathrm{Si}_{4}^{-}$, and $\mathrm{Au}_{6}^{-}$clusters are estimated to be $250 \mathrm{fs}, 1.2 \mathrm{ps}$, and about $1 \mathrm{~ns}$, respectively. These clusters show closed electronic shell structures and large gaps between the highest occupied molecular orbital and the lowest unoccupied molecular orbital in the anionic $\left(\mathrm{Al}_{13}^{-}\right)$or neutral $\left(\mathrm{Si}_{4}\right.$ and $\left.\mathrm{Au}_{6}\right)$ states. Thus the different behaviors of these clusters for the relaxation dynamics cannot be rationalized within a relaxation mechanism dominated by Auger-like electron-electron coupling. We suggest that for these small clusters, the major decay mechanism might be electron-phonon coupling.
\end{abstract}

PACS number(s): 78.47.+p, 33.60.Cv, 36.40.Vz

\section{INTRODUCTION}

Developments of femtosecond (fs) laser techniques have allowed us to study transition states in chemical reactions and relaxation dynamics of optically excited states in molecules and bulk materials. ${ }^{1-14}$ In particular, the time-resolved two-photon photoemission (TR-2PPE) spectroscopy opened new insights into the relaxation dynamics of solid surfaces.

When particle size decreases below several nanometers (nm), unexpected physical and chemical properties appear. Continuous band structures, which are characteristics of bulk metals or large metal particles, are changed into the discrete electronic levels with decreasing cluster size. Modification of electronic structures as a function of cluster size can be reflecte in the relaxation dynamics of optically excited states. In general, the relaxation process can be understood by competition of transition and screening effects. ${ }^{15}$ On one hand, when the density of state (DOS) of occupied as well as unoccupied states decreases with decreasing particle size, the relaxation of optically excited states by inelastic electronelectron scattering is hampered or even not allowed. Therefore the relaxation rates of excited states in nanoclusters should be much longer than those of the respective bulk metals. On the other hand, a hole state created by excitation of an electron can be screened by other electrons, hampering decay of the excited electron. Therefore a lower DOS can reduce the screening effects, which increases the relaxation rate.

To shed light on the relaxation dynamics of nanoclusters, TR-2PPE studies on mass-selected gas phase clusters have been carried out. For the $d$-metal clusters such as Pt, Pd, and $\mathrm{Ni}$ clusters consisting of less than seven atoms, the relaxation rates of the optically excited states were shown to be as fast as several tens to hundreds of fs, which are comparable to those of the respective bulk crystals. ${ }^{16-19}$ These results can be explained by relatively high DOS of unoccupied states within the pump photon energy above the highest occupied molecular orbital (HOMO) in these $d$-metal clusters. Even though DOS of the nanoclusters are lower than those of the bulk counterparts, the number of electronic states in a nanocluster is still sufficientl large to allow fast Auger-like electron-electron scattering, i.e., the averaged gaps between different electronic levels are much smaller compared to the primary excitation energy allowing energy distribution into the electronic degrees of freedom by cascades of inelastic scatterings. For "simple"-metal clusters, in which the valence electronic levels consist of $s / p$-orbitals, in contrast, indications for much slower relaxation rates were found: optically excited $\mathrm{Au}_{3}^{-}$can be decomposed into $\mathrm{Au}_{2}^{-}$and $\mathrm{Au}_{1}$, implying that the excited state of $\mathrm{Au}_{3}^{-}$relaxes on the time scale of several nanoseconds (ns). ${ }^{20}$ For Na-dimers, wave packet motions can be observed, also indicative of the existence of a very long-living excited state. ${ }^{21}$ For $\mathrm{Ag}_{8}$ clusters, the fluores cence phenomena are detected, which can be only observed when relaxation rates of optically excited states are of the order of ns. ${ }^{22}$ The much slower relaxation rates in these "simple"-metal clusters can be explained by their lower DOS with respect to those of the $d$-metals, which reduces the inelastic electron-electron scattering rate. Note that DOS of $d$-metal clusters (of the occupied and unoccupied states) are about two orders of magnitude larger than those of the $s p$-metal clusters with similar sizes. ${ }^{17}$ For the mass-selected clusters consisting of less than 10-15 atoms, decreased DOS reduces the relaxation rates implying that screening effects play a minor role in the relaxation dynamics.

Among various nanoclusters, the so-called magic clusters are of particular interest. Magic clusters are chemically inert and exhibit much higher stabilities with respect to nonmagic clusters. Magic clusters are not only of fundamental interest but also of technological importance, since they are promising candidates for building blocks of cluster materials, which are expected to be used in nano-devices. Magic clusters generally have closed electronic shell structures, ${ }^{23}$ high ionization potentials, and large gaps between the highest occupied molecular orbital (HOMO) and the lowest unoccupied molecular orbital (LUMO). Well-known examples for the magic clusters are $\mathrm{C}_{60},{ }^{24} \mathrm{Au}_{20},{ }^{25} \mathrm{Al}_{13}^{-},{ }^{26} \mathrm{Al}_{13} \mathrm{H},{ }^{27} \mathrm{Si}_{4},{ }^{28}$ and $\mathrm{Sb}_{4} \cdot{ }^{29}$ Due to the large HOMO-LUMO gaps, inelastic electronelectron scattering should be forbidden in magic clusters, yielding relatively long relaxation rates via electron-phonon couplings. In fact, the relaxation rate of an excited state in $\mathrm{C}_{60}$ was shown to be longer than a microsecond. ${ }^{30,31}$

In the present work, attempts were made to shed light on the relaxation dynamics of magic clusters. We measured relaxation rates of $\mathrm{Al}_{13}^{-}, \mathrm{Au}_{6}^{-}$, and $\mathrm{Si}_{4}^{-}$using TR-2PPE spectroscopy. $\mathrm{Al}_{13}$ is a magic cluster in the anionic state, ${ }^{26}$ whereas 


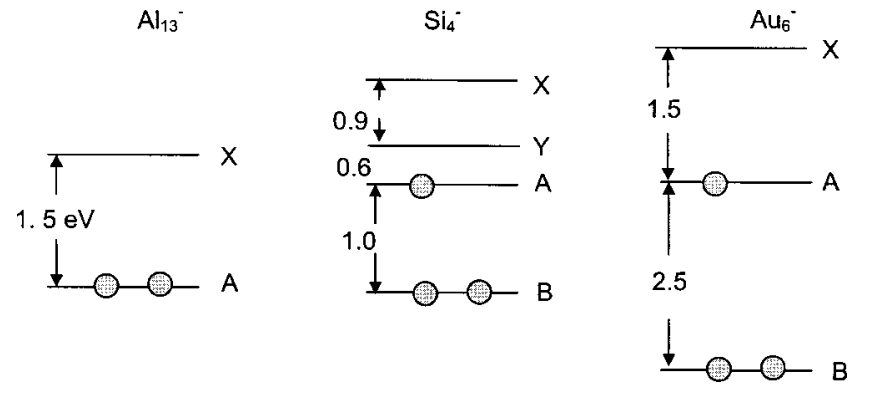

FIG. 1. Energy diagrams of the valence electronic structures of $\mathrm{Al}_{13}^{-}, \mathrm{Si}_{4}^{-}$, and $\mathrm{Au}_{6}^{-}$. The labels correspond to the electronic states in Figs. 2-4.

$\mathrm{Au}_{6}$ Ref. 32 and $\mathrm{Si}_{4}$ Ref. 28 show characteristics of magic clusters as neutrals. It has been demonstrated that an additional electron in a magic cluster hardly alters the cluster geometry and electronic levels. ${ }^{25}$ Therefore the energy gap between HOMO and the next occupied electronic level of the anion of a magic cluster is almost identical to the HOMOLUMO gap of the neutral counterpart (Fig. 1). Due to these large gaps as shown in Fig. 1, it is expected that the Augerlike electron-electron scattering cascade does not take place in $\mathrm{Au}_{6}^{-}$and $\mathrm{Si}_{4}^{-}$as well as $\mathrm{Al}_{13}^{-}$after the valence electrons are optically excited, i.e., analogous to the case of small $s p$-metal clusters mentioned above, very long relaxation rates are expected for all the three clusters. However, our results show that behaviors of these clusters for the relaxation dynamics are quite different. $\mathrm{For}^{-} \mathrm{Al}_{13}^{-}$, a decay time of about $260 \mathrm{fs}$ was found, whereas for $\mathrm{Si}_{4}^{-}$, the decay time of 1.2 picoseconds (ps) was determined. For $\mathrm{Au}_{6}^{-}$, the excited states can live for about $1 \mathrm{~ns}$. The origin of the dissimilar relaxation dynamics of the clusters with analogous electronic properties is discussed.

\section{EXPERIMENTAL DETAILS}

$\mathrm{Au}, \mathrm{Al}$, and $\mathrm{Si}$ clusters anions are produced by means of a pulsed arc cluster ion source (PACIS) and mass-selected using a time-of-fligh (ToF) mass spectrometer. ${ }^{33} \mathrm{~A}$ selected bunch of cluster anions is irradiated by two pulses generated in a femtosecond laser system. The cluster temperature is estimated to be room temperature. ${ }^{33}$ The firs pulse (pump pulse) has a photon energy of $1.5 \mathrm{eV}$ (red pulse, for $\mathrm{Al}_{13}^{-}$, $1.55 \mathrm{eV}$ ) and the second pulse (probe pulse) a photon energy of $3.05 \mathrm{eV}$ (blue pulse, for $\mathrm{Al}_{13}^{-}, 3.1 \mathrm{eV}$ ). The upper limit of the instrumental time resolution was estimated be about $150 \mathrm{fs}$. The pump pulse results in photoexcitation of a certain fraction of the anions in the bunch and the probe pulse detaches electrons from a fraction of the anions in the same bunch. The photon flu of both pulses has to be optimized to maximize the number of the species hit by a single photon of each pulse and to minimize the number of the species hit by two or more photons from the same pulse. For the pump pulse alone, this has been achieved by adjusting the photon flu gain to less than $10 \%$ with respect to photoelectron intensity created by two-photon process of the pump pulse. This procedure minimizes the "false" two-photon pump electron signal with respect to the "true" pump-probe signal. The

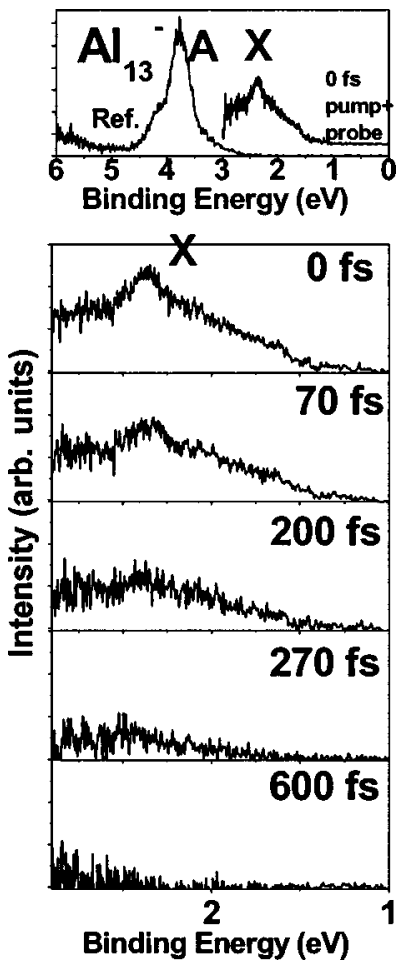

FIG. 2. A standard UPS spectrum (top trace) and TR-2PPE difference spectra of $\mathrm{Al}_{13}^{-}$are displayed. For comparison, the TR-2PPE spectrum at $0 \mathrm{fs}$ delay is inserted in the reference spectrum. The reference spectrum was taken using a photon energy of $6.4 \mathrm{eV}$.

same procedure has been applied to the adjustment of the photon flu of the probe pulse minimizing two-photon processes of the probe pulse alone, which can be easily detected by the appearance of electrons at higher kinetic energies. The photon flu of both pump and probe pulses have to be readjusted for different clusters because the cross sections of the corresponding processes may vary significantl as a function of cluster size.

\section{RESULTS AND DISCUSSIONS}

\section{A. Relaxation dynamics of $\mathbf{A l}_{13}^{-}$}

$\mathrm{Al}_{13}^{-}$is a magic cluster with an icosahedral geometry, having 40 valence electrons with a closed shell structure, and a HOMO-LUMO gap of $1.5 \mathrm{eV} .{ }^{26,34,35}$ Due to its high symmetry, the reference UPS spectrum of $\mathrm{Al}_{13}^{-}$recorded with $6.4 \mathrm{eV}$ photon energy shows a single peak at $3.8 \mathrm{eV}$ (Fig. 2, upper window, trace A). At a temperature of $300 \mathrm{~K}$, a single peak at $3.8 \mathrm{eV}$ is expected without additional features at lower binding energies, however, in our reference spectrum, an additional broad tail can be observed between 2.5 and $3.5 \mathrm{eV}$. We assign this tail to two-photon processes. The energy of the firs photon is thermalized within the duration of a nanosecond pulse and the second photon from the same pulse detaches an electron from this "hot" cluster. The reference UPS spectrum consists of the electron signals from the "cold" and "hot" clusters.

For $\mathrm{Al}_{13}^{-}$, a narrow peak (peak $\mathrm{X}$ in Fig. 2) appears at a binding energy corresponding to the LUMO overlapped with 


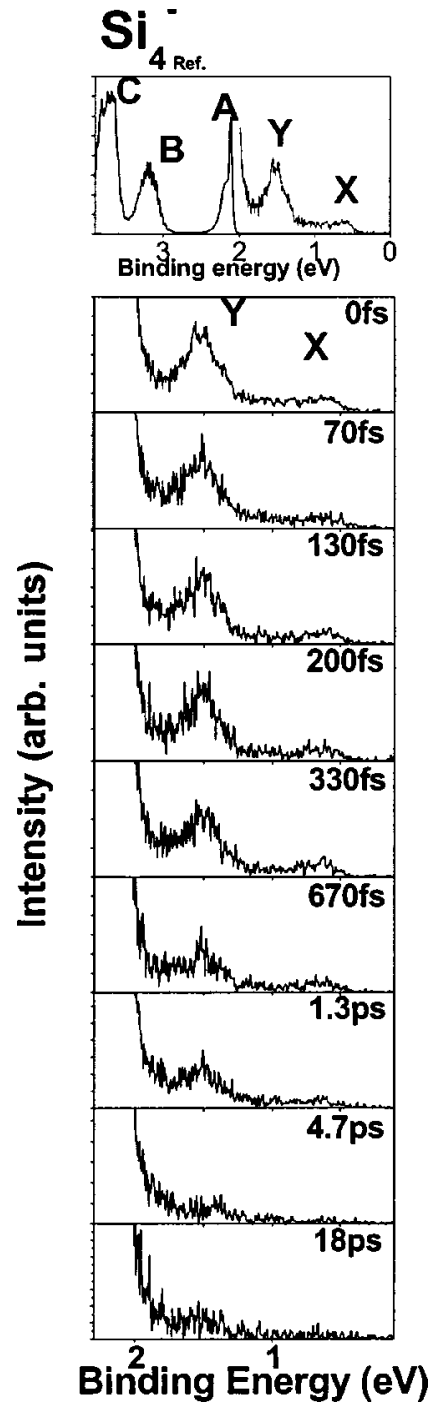

FIG. 3. A standard UPS spectrum and TR-2PPE spectra of $\mathrm{Si}_{4}^{-}$ are displayed. The reference spectrum was taken using a photon energy of $4.66 \mathrm{eV}$. For comparison, the TR-2PPE spectrum at $0 \mathrm{fs}$ delay is inserted in the reference spectrum.

a broad background signal in the energy range between 1.5 and $3 \mathrm{eV}$ for a pump-probe delay below $600 \mathrm{fs}$. The peak $\mathrm{X}$ in the pump/probe signal corresponds to a standard pump/ probe event, in which the pump pulse (photon energy $=1.55 \mathrm{eV}$ ) excites one electron from the HOMO to the LUMO, and a probe pulse (photon energy $=3.1 \mathrm{eV}$ ) detaches the excited electron. The broad background signal is produced by a two-photon event of the probe pulse (Fig. 2). Similar background features are observed even when the pump-pulse is switched-off, giving rise to an electron signal (not shown) extending up to a maximum kinetic energy of $2.4 \mathrm{eV}$ (binding energy $=0.6 \mathrm{eV}$ ). It is noteworthy that only broad features appear up to the binding energy of $0.6 \mathrm{eV}$ as a result of the two-photon process of the probe pulse without any distinct peaks. This could be rationalized by the electronphonon scattering process if it is sufficientl fast to allow thermalization of the energy of the firs photon within the duration of a probe pulse. Assuming an exponential decay of
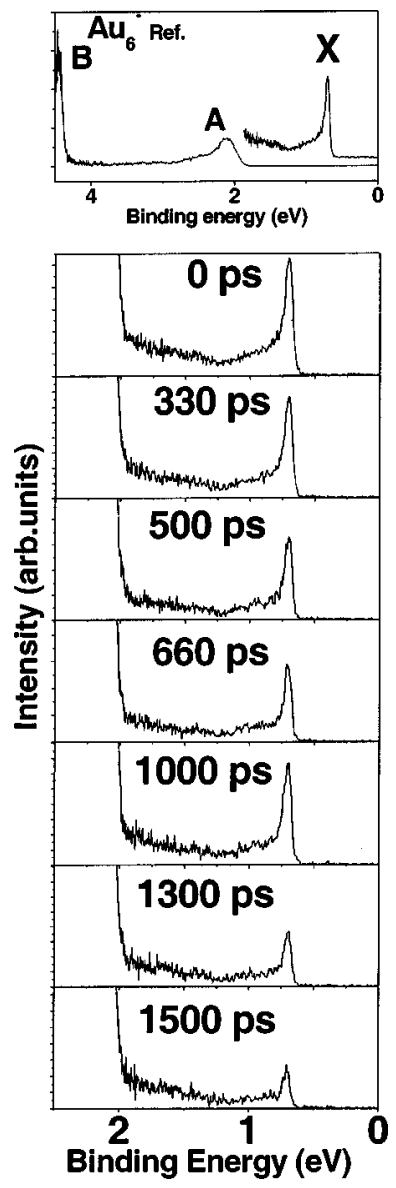

FIG. 4. A standard UPS spectrum and TR-2PPE spectra of $\mathrm{Au}_{6}^{-}$ are displayed. The reference spectrum was taken using a photon energy of $4.66 \mathrm{eV}$. For comparison, the TR-2PPE spectrum at 0 fs delay is inserted in the reference spectrum.

the excited state, the decay time of $\mathrm{Al}_{13}^{-}$is estimated to be about $260 \mathrm{fs}$, which is longer than those of Pt and Pd clusters consisting of less than seven atoms, however, comparable to that of $\mathrm{Ni}_{3}^{-} .{ }^{16-19}$ This result is quite surprising considering that the relaxation rate of $\mathrm{Al}_{13}^{-}$is expected to be much longer than those of the $d$-metal clusters as a consequence of the large HOMO-LUMO gap of $\mathrm{Al}_{13}^{-}$, which does not allow the inelastic electron-electron scattering, and results in the relaxation predominantly via the electron-phonon coupling.

\section{B. Relaxation dynamics of $\mathrm{Si}_{4}^{-}$}

$\mathrm{Si}_{4}$ has a rhombus structure with a HOMO-LUMO gap of about $1 \mathrm{eV} .{ }^{28}$ The vertical detachment energy (VDE) of $\mathrm{Si}_{4}$ is $2.2 \mathrm{eV} .{ }^{36} \mathrm{For}^{-} \mathrm{Si}_{4}^{-}$, the reference UPS spectrum shows a distinct peak at about $2.1 \mathrm{eV}$, followed by a broader peak at about $3.1 \mathrm{eV}$ (Fig. 2). The binding energy difference between peak $\mathrm{A}$ and $\mathrm{B}$ corresponds to the HOMO-LUMO gap of neutral $\mathrm{Si}_{4}$. Two broad features appear at the binding energy range below $2 \mathrm{eV}$ upon excitation by a pump pulse (X,Y in Fig. 3), which gradually disappear with increasing time interval between the pump and probe pulses. For $\mathrm{Si}_{4}^{-}$, the TR-2PPE results show a decay time of $1.2 \mathrm{ps}$, which is considerably longer than that of $\mathrm{Al}_{13}^{-}$, but still quite fast com- 
pared to those of the small $s p$-metal clusters mentioned in the Introduction. Again, due to the large gap between the HOMO and the next occupied orbital, the inelastic electronelectron coupling should be forbidden in a $\mathrm{Si}_{4}^{-}$cluster, and therefore a rather long relaxation rate in the order of ns is expected. However, our results are in contrast to this expectation.

\section{Relaxation dynamics of $\mathrm{Au}_{6}^{-}$}

$\mathrm{Au}_{6}$ as well as its anionic counterpart show planar structures and the HOMO-LUMO gap of $\mathrm{Au}_{6}$ amounts to about $2.5 \mathrm{eV},{ }^{32,37}$ which is the largest among all-known coinage metal clusters. TR-2PPE spectra of $\mathrm{Au}_{6}^{-}$are illustrated in Fig. 4. The set of the spectra corresponds to the red/blue excitation, i.e. $\mathrm{Au}_{6}^{-}$clusters are firs excited by the $1.5 \mathrm{eV}$ photon, and then probed using the $3.05 \mathrm{eV}$ photon. A narrow single peak appears at the binding energy of $0.8 \mathrm{eV}$, which is still present for the time interval of several hundreds of picoseconds between the pump and probe pulses. Assuming an exponential decay of the optically excited states in $\mathrm{Au}_{6}^{-}$, we estimate the lifetimes of the two different excited states observed in the red/blue experiment to be $1.2 \mathrm{~ns}$. These relatively long relaxation rates of the excited states can be explained by a large gap between the HOMO and the next occupied orbital in $\mathrm{Au}_{6}^{-}(2.5 \mathrm{eV})$, which should forbid the electron-electron coupling and only allows relaxation of the excited state via the conversion of the excess energy of the electron into the nuclear motions of the cluster (electronphonon coupling).

\section{Comparison of the relaxation dynamics of three magic clusters}

We have shown that $\mathrm{Al}_{13}^{-}, \mathrm{Si}_{4}^{-}$, and $\mathrm{Au}_{6}^{-}$behave differently in the relaxation dynamics. As mentioned in the Introduction, in the case of metal bulk crystals and $d$-metal clusters, numbers of unoccupied electronic levels within the optical excitation energy (pump energy) above the HOMO of the ground state are sufficientl large. Thus the excess energy of the electron excited by the pump photon can experience fast dissipation via inelastic electron-electron scatterings, yielding relaxation times of the order of tens to hundreds of fs. For those clusters studied in the present work, in contrast, this Auger-like inelastic electron-electron scattering is forbidden since there is no unoccupied electronic levels above the $\mathrm{HOMO}$ (for $\mathrm{Al}_{13}^{-}$) or the orbitals next to the HOMO (For $\mathrm{Si}_{4}^{-}$, $\mathrm{Au}_{6}^{-}$) within the pump photon energy and therefore, the decay time should be limited by the electron-phonon coupling. In the case of $\mathrm{Si}_{4}^{-}$, one may argue that an electron in the electronic level next to the HOMO (level B in Fig. 2) is excited by the pump pulse, yielding the peak $\mathrm{Y}$ in the pump/ probe signal. Then, two electrons occupy the upper states (A and $\mathrm{Y}$ in Fig. 2), and a hole is created in the lower state, which can lead to two-electron Auger processes. However, this kind of Auger process in $\mathrm{Si}_{4}^{-}$cannot result in the emission of an electron outside the vacuum level. Note that the relaxation of an electron from the upper level (A or Y) into the lower level (B) can produce an excess energy of $1.5 \mathrm{eV}$ or less, which is not sufficien to bring an electron in $\mathrm{Y}$ or A above the vacuum level. If this kind of two-electron Auger process takes place, additional features should be created at lower binding energies of the pump/probe spectra, as the intensity of the peak $\mathrm{Y}$ is reduced. However, indications for this kind of two-electron Auger-process were not observed in Fig. 2. That the peak Y and X appear and disappear almost simultaneously with increasing pump/probe time interval are not consistent with this two-electron Auger process. Consequently, electron-electron coupling cannot be the dominant relaxation mechanism for these three clusters.

In general, relaxation via electron-phonon coupling takes place much slower compared to those of the inelastic electron-electron scattering. Relaxation times amount to several hundreds of ps to several ns when the conversion of the excess energy into the nuclear motions becomes the dominant relaxation mechanism in molecules or clusters. In contrast to this generally accepted view, our results from three magic clusters imply that for small nanoclusters the electronphonon coupling constant can vary from several hundreds of femtoseconds to one nanosecond depending on the cluster composition and geometry (Figs. 2-4). It is important to mention that in our recent experiments for the relaxation dynamics of $\mathrm{Al}$ clusters consisting of 6-15 atoms, we could not fin any correlation between relaxation rates and valence electron structures of the Al clusters, also implying that the relaxation of these clusters are governed by the electronphonon coupling. ${ }^{38}$ Moreover, as mentioned above, an indication for the fast electron-phonon scattering was observed as a broad background signal in the TR-2PPE spectra of $\mathrm{Al}_{13}^{-}$ in Fig. 2. Other mechanisms such as relaxation via the collective excitation of electrons can be also considered, however, the plasmon energy of $\mathrm{Al}$ lies at above $10 \mathrm{eV},{ }^{39}$ which is too high to rationalize the electron-plasmon coupling. Further theoretical studies can shed light on the relaxation mechanisms of the clusters.

\section{CONCLUSION}

In summary, relaxation rates of the optically excited states in $\mathrm{Al}_{13}^{-}, \mathrm{Si}_{4}^{-}$, and $\mathrm{Au}_{6}^{-}$clusters were determined to be $260 \mathrm{fs}$, $1.2 \mathrm{ps}$, and about $1 \mathrm{~ns}$, respectively. Since the electronelectron coupling should be forbidden in a magic cluster or its anionic counterpart, relatively fast relaxation dynamics of $\mathrm{Al}_{13}^{-}$and $\mathrm{Si}_{4}^{-}$cannot be rationalized within a simple scheme, in which the inelastic electron-electron scattering is the dominant relaxation mechanism. One possible explanation for our results is that the electron-phonon scattering is the major relaxation mechanism.

\section{ACKNOWLEDGMENT}

We aknowledge DFG (Deutsche Forschungsgemeinschaft) for the financia support. 
*Corresponding author. Email address: young.kim@unikonstanz.de

${ }^{1}$ A. H. Zewail, Femtochemisty (World Scientific Singapore, 1994), Vols I and II.

${ }^{2}$ H. Petek and S. Ogawa, Prog. Surf. Sci. 56, 239 (1997).

${ }^{3}$ C. Voisin, D. Christofilos N. Del Fatti, F. Vallee, B. Prevel, E. Cottancin, J. Lerme, M. Pellarin, and M. Broyer, Phys. Rev. Lett. 85, 2200 (2000).

${ }^{4}$ S. Vajda, C. Lupulescu, A. Merli, F. Budzyn, and L. Wöste, Phys. Rev. Lett. 89, 213404 (2002).

${ }^{5}$ D. M. Neumark, Science 272, 1446 (1996).

${ }^{6}$ J. M. Weber, J. Kim, E. A. Woronowicz, C. H. Weddle, I. Becker, O. Cheshnovsky, and M. A. Johnson, Chem. Phys. Lett. 339, 337 (2001).

${ }^{7}$ R. Schlipper, R. Kusche, B. von Issendorff, and H. Haberland, Phys. Rev. Lett. 80, 1194 (1998).

${ }^{8}$ Femtosecond Chemistry, edited by J. Manz and L. Wöste (VCHVerlagsgesellschaft, Wahnheim, Germany, 1995), Vols. I and II.

${ }^{9}$ S. Pawlik, M. Bauer, and M. Aschliemann, Surf. Sci. 377-379, 206 (1997).

${ }^{10}$ T. Hertel, E. Knoesel, M. Wolf, and G. Ertl, Phys. Rev. Lett. 76, 535 (1996).

${ }^{11}$ E. Knoesel, A. Hotzel, T. Hertel, M. Wolf, and G. Ertl, Surf. Sci. 368, 76 (1996)

${ }^{12}$ M. Wolf, E. Knoesel, and T. Hertel, Phys. Rev. B 54, R5295 (1996).

${ }^{13}$ P. Farmanara, H. H. Ritze, V. Stert, W. Radloff, and I. V. Hertel, J. Chem. Phys. 116, 1443 (2002).

${ }^{14}$ M. Wollenhaupt, A. Assion, D. Liese, C. S. Sarpe-Tudoran, T. Baumert, S. Zamith, M. A. Bouchene, B. Girard, A. Flettner, U. Weichmann, and G. Gerber, Phys. Rev. Lett. 89, 173001 (2002).

${ }^{15}$ P. M. Echenique, J. M. Pitarke, E. V. Chulkov, and A. Rubio, Chem. Phys. 251, 1 (2000).

${ }^{16}$ N. Pontius, P.-S. Bechthold, M. Neeb, and W. Eberhardt, Phys. Rev. Lett. 84, 1132 (2000).

${ }^{17}$ N. Pontius, M. Neeb, W. Eberhardt, G. Lüttgens, and P. S. Bechthold, Phys. Rev. B 67, 035425 (2003).

${ }^{18}$ N. Pontius, G. Lüttgens, P. S. Bechthold, M. Neeb, and W. Eberhardt, J. Chem. Phys. 115, 10479 (2001).
${ }^{19}$ N. Pontius, P. S. Bechthold, M. Neeb, and W. Eberhardt, J. Electron Spectrosc. Relat. Phenom. 114-116, 163 (2001).

${ }^{20}$ G. Ganteför, S. Kraus, and W. Eberhardt, J. Electron Spectrosc. Relat. Phenom. 88-91, 35 (1998).

${ }^{21}$ A. Assion, M. Geisler, J. Helbing, V. Seyfried, and T. Baumert, Phys. Rev. A 54, R4605 (1996).

${ }^{22}$ C. Felix, C. Sieber, W. Harbich, J. Buttet, I. Rabin, W. Schulze, and G. Ertl, Phys. Rev. Lett. 86, 2992 (2001).

${ }^{23}$ W. A. de Heer, Rev. Mod. Phys. 65, 611 (1993).

${ }^{24}$ H. W. Kroto, J. R. Heath, S. C. O'Brien, R. F. Curl, and R. E. Smalley, Nature (London) 318, 162 (1985).

${ }^{25}$ J. Li, X. Li, H.-J. Zhai, and L.-S. Wang, Science 299, 864 (2003).

${ }^{26}$ X.-B. Wang and L.-S. Wang, Phys. Rev. Lett. 81, 1909 (1998).

${ }^{27}$ S. Buckart, N. Blessing, B. Klipps, J. Müller, G. Ganteför, and G. Seifert, Chem. Phys. Lett. 301, 546 (1999).

${ }^{28}$ M. Grass, D. Fischer, M. Mathe, G. Ganteför, and P. Nielaba, Appl. Phys. Lett. 81, 3810 (2002).

${ }^{29}$ T. M. Bernhardt, B. Stegemann, B. Kaiser, and K. Rademann, Angew. Chem., Int. Ed. 42, 199 (2003).

${ }^{30}$ J. W. Arbogast, A. P. Darmanyan, C. S. Foote, Y. Rubin, F. N. Diederich, M. M. Alvarez, S. J. Anz, and R. L. Whetten, J. Chem. Phys. 95, 11 (1995).

${ }^{31}$ R. W. Haufler L.-S. Wang, L. P. F. Chinabte, C. Jin, J. Conceicao, Y. Chai, and R. E. Smalley, Chem. Phys. Lett. 179, 449 (1991).

${ }^{32}$ K. J. Taylor, C. L. Pettiette-Hall, O. Cheshnovsky, and R. E. Smalley, J. Chem. Phys. 96, 3319 (1992).

${ }^{33}$ C.-Y. Cha, G. Ganteför, and W. Eberhardt, Rev. Sci. Instrum. 63, 5661 (1992).

${ }^{34}$ S. N. Khanna, and P. Jena, Chem. Phys. Lett. 218, 383 (1994).

${ }^{35}$ J. Akola, M. Manninen, H. Häkkinen, U. Landman, X. Li, and L.-S. Wang, Phys. Rev. B 60, R11 297 (1999).

${ }^{36}$ J. Müller, B. Liu, A. A. Shvartsburg, S. Ogut, J. R. Chelikowsky, K. W. Michael Siu, K.-M. Ho, and G. Ganteför, Phys. Rev. Lett. 85, 1666 (2000).

${ }^{37}$ H. Häkkinen, M. Moseler, and U. Landman, Phys. Rev. Lett. 89, 033401 (2002).

${ }^{38}$ P. Gerhardt, M. Niemietz, Y. D. Kim, and G. Ganteför, Chem. Phys. Lett. 382, 454 (2003).

${ }^{39}$ M. Rocca, Surf. Sci. Rep. 22, 1 (1995). 